\title{
Anti-Glypican 3-scFvGC33-CAR-expressing T Lymphocytes
}

National Cancer Institute

\section{Source}

National Cancer Institute. Anti-Glypican 3-sCFvGC33-CAR-expressing T Lymphocytes. NCI

Thesaurus. Code C132989.

A preparation of autologous T-lymphocytes that have been genetically modified to express a chimeric antigen receptor (CAR) containing a single chain variable fragment (scFv) derived from the anti-glypican-3 (GPC3) monoclonal antibody GC33 (scFvGC33), with potential immunostimulating and antineoplastic activities. Upon administration, antiGPC3-scFvGC33-CAR autologous T-lymphocytes specifically target and bind to GPC3expressing tumor cells, resulting in tumor cell lysis. GPC3, a heparan sulfate proteoglycan and a member of the glypican family, is overexpressed on certain tumor cell types while minimally expressed in normal, healthy cells.. GPC3 plays an important role in cellular proliferation and differentiation. 